APS/123-QED

\title{
Elliptical flow and isospin effects in heavy-ion collisions at intermediate energies
}

\author{
Sanjeev Kumar and Suneel Kumar* \\ School of Physics and Material Science, \\ Thapar University, \\ Patiala-147004, Punjab (India) \\ Rajeev K. Puri \\ Department of Physics, Panjab University, \\ Chandigarh-160014 (India)
}

(Dated: November 14, 2018)

\begin{abstract}
The elliptical flow of fragments is studied for different systems at incident energies between 50 and $1000 \mathrm{MeV} /$ nucleon using Isospin-dependent Quantum Molecular Dynamics (IQMD) Model. Our findings reveal that elliptical flow shows a transition from positive (in-plane) to negative (out-of-plane) value in the mid-rapidity region at certain incident energy, known as transition energy. This transition energy is found to depend on the model ingredients, size of the fragments, composite mass of the reacting system as well as on the impact parameter of the reaction. A reasonable agreement is observed for the excitation function of elliptical flow between the data and our calculations. Interestingly, the transition energy is found to exhibit a power law mass dependence.
\end{abstract}

PACS numbers: 25.70.-z, 25.75.Ld, 21.65.Ef

*Electronic address: suneel.kumar@thapar.edu 


\section{INTRODUCTION}

The information about the nature of equation of state is still one of the burning topic of present day nuclear physics research in general and heavy-ion collisions in particular. A quite good progress has been made in the recent years in determining the nuclear equation of state from heavy-ion reactions [1, 2]. Among different observables, collective flow enjoys a special status. This is due to its sensitive response to the model ingredients that define equation of state. A lot of theoretical and experimental efforts have been made in studying the collective flow in heavy-ion collisions [3-9]. This collective motion of the particles in heavy-ion collision can be studied via directed and elliptical flows. The directed flow, which measures the collective motion of the particles in the reaction plane, has been studied extensively at BEVALAC, SIS and AGS energies [10]. This flow is reported to diminish at higher incident energies due to the large beam rapidity. Therefore, elliptical flow [11] is much more suited at these incident energies. The elliptical flow describes the eccentricity of an ellipse like distribution. Quantitatively, it is the difference between the major and minor axis. The orientation of the major axis is confined to azimuthal angle $\phi$ or $\phi+\frac{\pi}{2}$ for ellipse like distribution. The major axis lies within the reaction plane for $\phi$; while $\phi+\frac{\pi}{2}$ indicates that the orientation of the ellipse is perpendicular to the reaction plane, which is the case for squeeze out flow and may be expected at mid rapidity [12]. Therefore, the elliptical flow is defined by the second order Fourier coefficient from the azimuthal distribution of detected particles at mid rapidity. Mathematically,

$$
\frac{d N}{d \phi}=p_{0}\left(1+2 v_{1} \operatorname{Cos} \phi+2 v_{2} \operatorname{Cos} 2 \phi\right)
$$

Here $\phi$ is the azimuthal angle between the transverse momentum of the particle and reaction plane. The positive value of the elliptical flow $<\operatorname{Cos} 2 \phi>$ reflects an in-plane emission, whereas, out-of plane emission is reflected by its negative value. The reason for the anisotropic flow is orthogonal asymmetry in the configuration space (non-central collisions) and re-scattering. In the case of elliptical flow, the initial "ellipticity" of the overlap zone is usually characterized by a quantity $\epsilon=\frac{\left(<y^{2}-x^{2}>\right)}{\left(<y^{2}+x^{2}>\right)}$, assuming the reaction plane being xz. As the system expands, spatial anisotropy decreases. From the above discussion, it is clear that the second order flow (elliptical flow) is better candidate for determining the nuclear equation of state compared to first order sideward flow (directed flow). 
In recent years, several experimental groups have measure the elliptical flow. The FOPI, INDRA and PLASTIC BALL collaborations [4, 5] are actively involved in measuring the excitation function of elliptical flow from Fermi energies to relativistic energies. In most of

these studies, ${ }_{79} A u^{197}+{ }_{79} A u^{197}$ reaction has been taken [4, 5]. Interestingly, a change in the elliptical flow was reported from positive to negative values around $100 \mathrm{MeV} / \mathrm{nucleon}$. Both the mean field and two-body binary collisions play an important role in this energy domain. The mean field is supposed to play a dominant role at low incident energies. The binary collisions starts dominating the physics gradually. A detailed study of the excitation function of elliptical flow in entire energy region can provide a useful information about the nucleon-nucleon interactions related to the nuclear equation of state.

As discussed above, lots of attempts have already been made in the literature to explore different aspects of directed sideward flow. In this paper, we attempt to study the different aspects of elliptical flow $v_{2}$.

For the present study, Isospin-dependent Quantum Molecular Dynamics (IQMD) model is used to generate the phase space of nucleons. The article is organized as follow: we discuss the model briefly in section-II. The results are discussed in section-III and we summarize the results in section-IV.

\section{ISOSPIN-DEPENDENT QUANTUM MOLECULAR DYNAMICS (IQMD) MODEL}

The isospin-dependent quantum molecular dynamics (IQMD) [13] model treats different charge states of nucleons, deltas and pions explicitly[13], as inherited from the VUU model [14]. The IQMD model has been used successfully for the analysis of large number of observables from low to relativistic energies. The isospin degree of freedom enters into the calculations via symmetry potential, cross-sections and Coulomb interaction[14]. The details about the elastic and inelastic cross-sections for proton-proton and neutron-neutron collisions can be found in Ref.[13].

In this model, baryons are represented by Gaussian-shaped density distributions

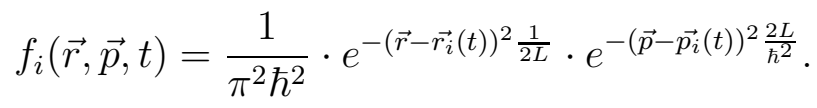


Nucleons are initialized in a sphere with radius $R=1.12 A^{1 / 3} \mathrm{fm}$, in accordance with the liquid drop model. Each nucleon occupies a volume of $h^{3}$, so that phase space is uniformly filled. The initial momenta are randomly chosen between 0 and Fermi momentum $\left(p_{F}\right)$. The nucleons of target and projectile interact via two and three-body Skyrme forces, Yukawa potential, Coloumb interactions and momentum-dependent interactions. In addition to the use of explicit charge states of all baryons and mesons a symmetry potential between protons and neutrons corresponding to the Bethe- Weizsacker mass formula has been included.

The hadrons propagate using Hamilton equations of motion:

$$
\frac{d \overrightarrow{r_{i}}}{d t}=\frac{d\langle H\rangle}{d \overrightarrow{p_{i}}} ; \frac{d \overrightarrow{p_{i}}}{d t}=-\frac{d\langle H\rangle}{d \overrightarrow{r_{i}}}
$$

with

$$
\begin{aligned}
\langle H\rangle= & \langle T\rangle+\langle V\rangle \\
= & \sum_{i} \frac{p_{i}^{2}}{2 m_{i}}+\sum_{i} \sum_{j>i} \int f_{i}(\vec{r}, \vec{p}, t) V^{i j}\left(\vec{r}^{\prime}, \vec{r}\right) \\
& \times f_{j}\left(\vec{r}^{\prime}, \vec{p}^{\prime}, t\right) d \vec{r} d \vec{r}^{\prime} d \vec{p} d \vec{p}^{\prime} .
\end{aligned}
$$

The baryon-baryon potential $V^{i j}$, in the above relation, reads as:

$$
\begin{aligned}
V^{i j}\left(\vec{r}^{\prime}-\vec{r}\right)= & V_{\text {Skyrme }}^{i j}+V_{\text {Yukawa }}^{i j}+V_{\text {Coul }}^{i j}+V_{m d i}^{i j}+V_{\text {sym }}^{i j} \\
= & \left(t_{1} \delta\left(\vec{r}^{\prime}-\vec{r}\right)+t_{2} \delta\left(\vec{r}^{\prime}-\vec{r}\right) \rho^{\gamma-1}\left(\frac{\vec{r}^{\prime}+\vec{r}}{2}\right)\right) \\
& +t_{3} \frac{\exp \left(\left|\vec{r}^{\prime}-\vec{r}\right| / \mu\right)}{\left(\left|\vec{r}^{\prime}-\vec{r}\right| / \mu\right)}+\frac{Z_{i} Z_{j} e^{2}}{\left|\vec{r}^{\prime}-\vec{r}\right|} \\
& +t_{4} \ln ^{2}\left[t_{5}\left(\vec{p}_{i}^{\prime}-\vec{p}\right)^{2}+1\right] \delta\left(\vec{r}^{\prime}-\vec{r}\right) \\
& +t_{6} \frac{1}{\varrho_{0}} T_{3}^{i} T_{3}^{j} \delta\left(\vec{r}_{i}^{\prime}-\vec{r}_{j}\right) .
\end{aligned}
$$

Here $Z_{i}$ and $Z_{j}$ denote the charges of $i^{t h}$ and $j^{t h}$ baryon, and $T_{3}^{i}, T_{3}^{j}$ are their respective $T_{3}$ components (i.e. $1 / 2$ for protons and $-1 / 2$ for neutrons). Meson potential consists of Coulomb interaction only. The parameters $\mu$ and $t_{1}, \ldots ., t_{6}$ are adjusted to the real part of the nucleonic optical potential. For the density dependence of nucleon optical potential, standard Skyrme-type parameterization is employed. The momentum dependence $V_{m d i}^{i j}$ of the N-N interactions, which may optionally be used in IQMD, is fitted to experimental data in the real part of nucleon optical potential. The choice of equation of state (or compressibility) is still controversial one. Many studies advocate softer matter, whereas, 
much more believe the matter to be harder in nature [14, 15]. As noted [16], elliptical flow is unaffected by the choice of equation of state. For the present analysis, a hard $(\mathrm{H})$ and hard momentum dependent (HMD) equation of state, has been employed alongwith standard energy dependent cross-section.

\section{RESULTS AND DISCUSSION}

We, here, perform a complete systematic study for the mass range between 80 and 394 units and over full range of the impact parameter. We here simulate the reactions of ${ }_{20} \mathrm{Ca}^{40}+{ }_{20} \mathrm{Ca}^{40},{ }_{28} N i^{58}+{ }_{28} N i^{58},{ }_{41} N b^{93}+{ }_{41} N b^{93},{ }_{54} X e^{131}+{ }_{54} X e^{131}$ and ${ }_{79} A u^{197}+{ }_{79} A u^{197}$ at incident energies between 50 and $1000 \mathrm{MeV} /$ nucleon. In addition, the reactions of ${ }_{40} Z r^{96}+{ }_{40} Z r^{96}$ and ${ }_{44} R u^{96}+{ }_{44} R u^{96}$ are also simulated to check the isospin effects explicitly. As noted in Ref.[17], the relativistic effects do not play role at these incident energies and the intensity of sub-threshold particle production is very small. The phase space generated by the IQMD model has been analyzed using the minimum spanning tree (MST) [2, 18] method. The MST method binds two nucleons in a fragment if their distance is less than $4 \mathrm{fm}$. In recent years, several improvements have also been suggested [19]. One of the improvements is to also imply momentum cut of the order of Fermi momentum. This method is dubbed as MSTM method. The entire calculations are performed at $t=200$ $\mathrm{fm} / \mathrm{c}$. This time is chosen by keeping in view the saturation of the collective flow [8].

The elliptical flow is defined as the average difference between the square of the $\mathrm{x}$ and $\mathrm{y}$ components of the particle's transverse momentum. Mathematically, it can be written as:

$$
v_{2}=\left\langle\frac{p_{x}^{2}-p_{y}^{2}}{p_{x}^{2}+p_{y}^{2}}\right\rangle,
$$

where $p_{x}$ and $p_{y}$ are the $\mathrm{x}$ and $\mathrm{y}$ components of the momentum. The $p_{x}$ is in the reaction plane, while, $p_{y}$ is perpendicular to the reaction plane.

A positive value of the elliptical flow describes the eccentricity of an ellipse like distribution and indicates in-plane enhancement of the particle emission i.e. a rotational behavior. On the other hand, a negative value of $v_{2}$ shows the squeeze out effects perpendicular to the reaction plane. Obviously, zero value corresponds to an isotropic distribution in the transverse plane. The $v_{2}$ is generally extracted from the mid rapidity region. The parti- 


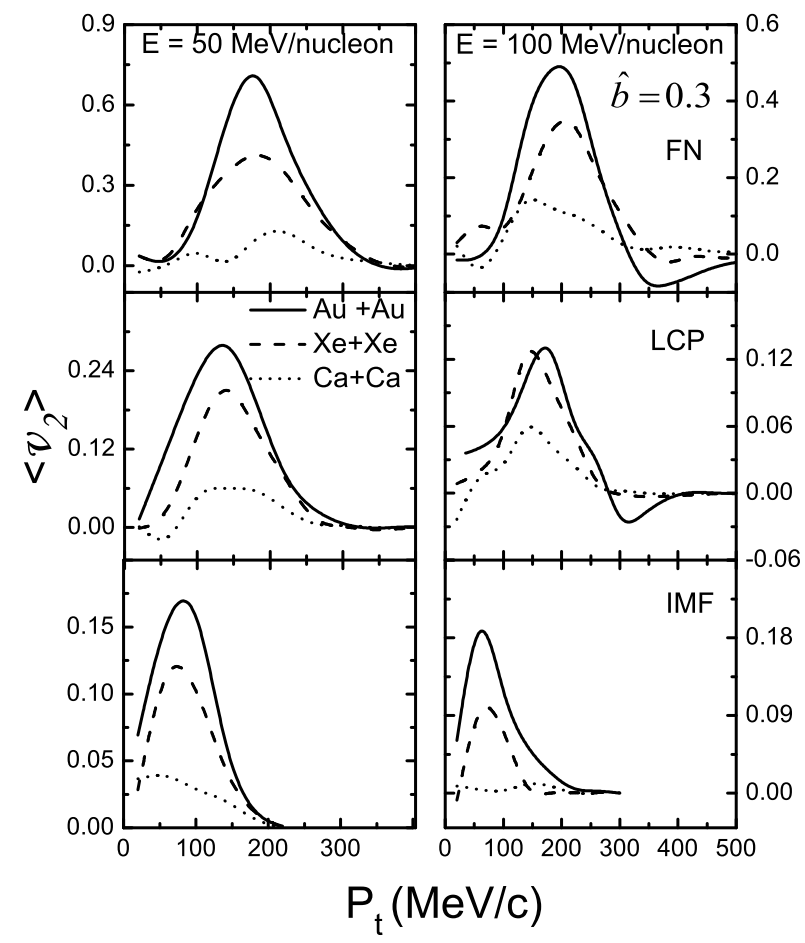

FIG. 1: The transverse momentum dependence of the elliptical flow, summed over entire rapidity distribution, at $\hat{b}=0.3$ for different symmetric reactions at 50 (left) and $100 \mathrm{MeV} /$ nucleon (right) respectively. The top, middle and bottom panels are representing the free nucleons (FN), light charged particles (LCP's) and intermediate mass fragments (IMF's), respectively.

cles corresponding to $\left(Y_{c . m .} / Y_{\text {beam }}>0.1\right)$ has been defined as projectile like $(\mathrm{PL})$, whereas, $\left(Y_{\text {c.m. }} / Y_{\text {beam }}<-0.1\right)$ constitutes the target like $(\mathrm{TL})$ particles.

In Fig,1, the final state elliptical flow is displayed for the free particles (upper panel), light charged particles (LCP's) $[2 \leq A \leq 4]$ (middle), and intermediate mass fragments (IMF's) $\left[5 \leq A \leq A_{t o t} / 6\right]$ (lower panel) as a function of transverse momentum $\left(P_{t}\right)$. A Gaussian type behavior is observed in all cases. Note that this elliptical flow is integrated over entire rapidity range. This Gaussian type behavior is quite similar to the one obtained by Colona and Toro et al. [20]. One sees that elliptical flow is positive in the whole range of $P_{t}$. The collective rotation is one of the main mechanism to induce the positive elliptical flow [21]. It is also evident from the figure that the peaks of the Gaussian shifts toward lower values of $P_{t}$ for heavier fragments. This is due to the fact that the free and light charged particles feel 


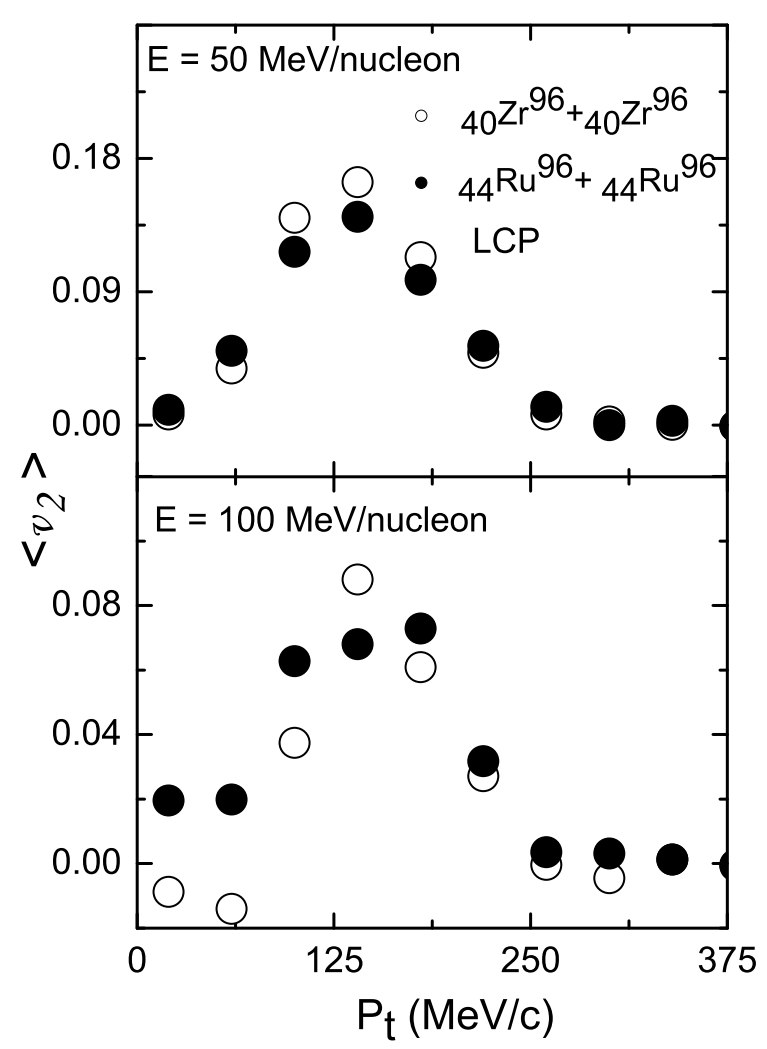

FIG. 2: The transverse momentum dependence of the elliptical flow, summed over entire rapidity distribution, for LCP's at 50 (top) and $100 \mathrm{MeV} /$ nucleon (bottom), respectively. The reactions under study having same mass number and different atomic number. The reactions are analyzed with MSTM algorithm.

the mean field directly, while heavy fragments have weaker sensitivity [22]. Furthermore, the peak values of $v_{2}$ for the free nucleons and LCP's at $50 \mathrm{MeV} /$ nucleon is $0.70,0.411$, 0.126 and $0.27,0.20,0.059$ for the reactions of ${ }_{79} A u^{197}+{ }_{79} A u^{197},{ }_{54} X e^{131}+{ }_{54} X e^{131}$ and ${ }_{20} \mathrm{Ca}^{40}+{ }_{20} \mathrm{Ca}^{40}$, respectively and the corresponding ratios are $\approx 5.0,3.3$ and 1 . The mass ratio of these reactions is $4.93,3.27$ and 1 , whereas, $N / Z$ ratio is $1.49,1.42$ and 1 . The $v_{2}$ ratios are in closer agreement with the system mass ratios. The results, however, are different at $\mathrm{E}=100 \mathrm{MeV} /$ nucleon. Note that the peak values for the free nucleon are 0.48 , 0.34, 0.134 and for LCP's numbers, are 0.132, 0.125, 0.058. Their corresponding ratios are $\approx 2.92,2.36$ and 1, indicating a clear deviation from the mass ratio.

To further strengthen our interpretation of the estimated $v_{2}$ ratios, we display in Fig,2, the reactions of ${ }_{40} Z r^{96}+{ }_{40} Z r^{96}$ and ${ }_{44} R u^{96}+{ }_{44} R u^{96}$ under the same conditions for 


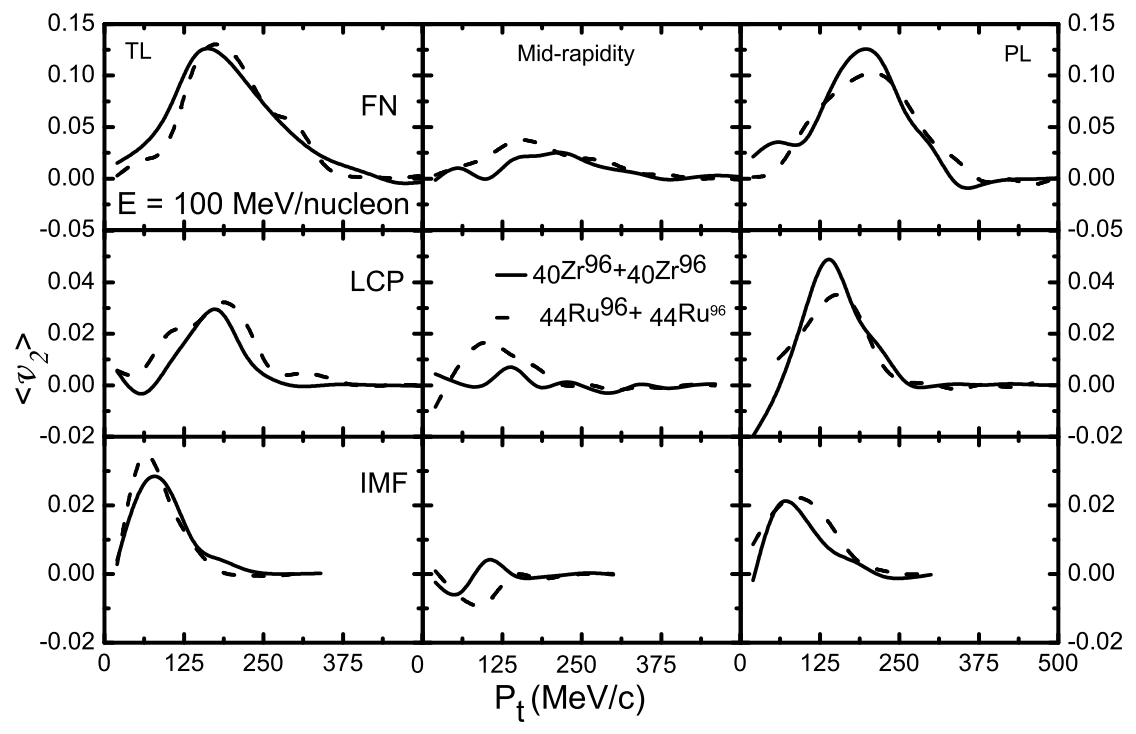

FIG. 3: The transverse momentum dependence of the elliptical flow at $E=100 \mathrm{MeV} /$ nucleon for the reactions displayed in Fig,2, The left, middle and right panels are representing target-like, midrapidity and projectile like distributions, respectively, while, top, middle and and bottom panels have same meaning as that of Fig. 1. The reactions are analyzed with MST algorithm.

LCP's. These reactions are analyzed within MST method with momentum cut. Interestingly, the $N / Z$ effect is more visible at $\mathrm{E}=100 \mathrm{MeV} /$ nucleon, indicating that this difference is not due to the mass dependence alone, but is due to isospin effect also. Our findings are also supported by Zhang et al. [23], where they showed that neutron-rich system exhibits weaker squeeze-out flow. At low incident energy (say $50 \mathrm{MeV} /$ nucleon), binary collisions are rare, therefore isospin in the mean field does not play role. On the other hand, around $100 \mathrm{MeV} /$ nucleon, both isospin of the mean field and binary collisions contribute, making isospin maximum. At higher incident energies, the role of mean field reduces. This situation is similar to the intermediate mass fragments, where maximum value is obtained around 100 $\mathrm{MeV} /$ nucleon [24].

To further understand the origin of this isospin effect, the transverse momentum dependence of elliptical flow for target-like, mid-rapidity and projectile-like distributions is displayed in Fig $[3$. From the figure, we see that isospin effect originates from the mid-rapidity region or in other words from the participant zone. It is also clear that the isospin effects are stronger 


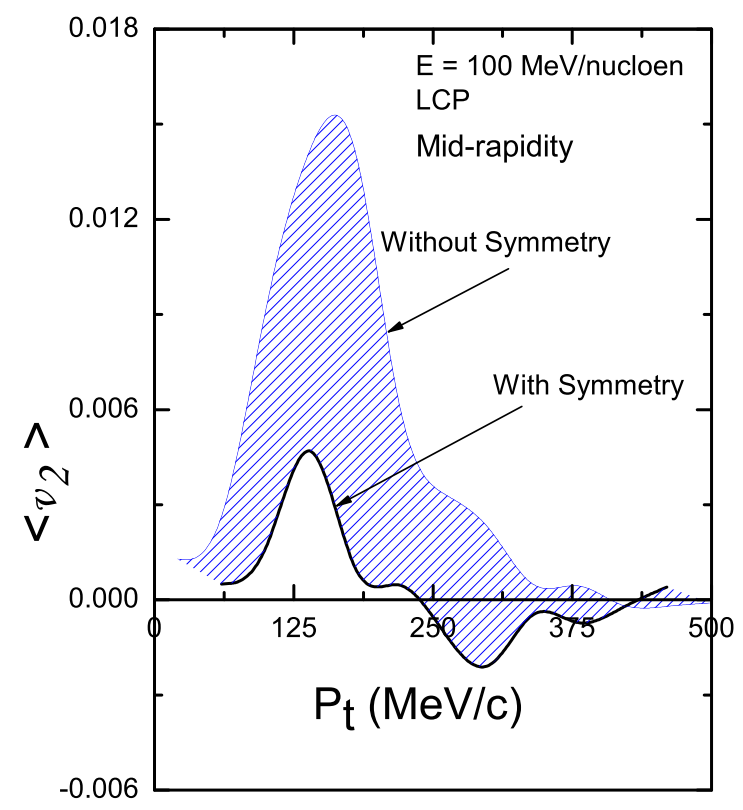

FIG. 4: The transverse momentum dependence of elliptical flow for LCP's in the mid-rapidity region at $\mathrm{E}=100 \mathrm{MeV} /$ nucleon. The panel is exhibiting the effect of symmetry energy on the ${ }_{40} Z r^{96}+{ }_{40} Z r^{96}$ reaction.

for LCP's compared to other fragments. This is due to the fact that heavier fragments have weak sensitivity towards mean field [22].

In Fig, 4, we display the transverse momentum dependence of elliptical flow for LCP's in the mid-rapidity region with and without symmetry energy. The effect of symmetry energy is clearly visible in the figure. This is in agreement with the findings of Chen et al. [25], where it was concluded that light clusters production acts as a probe for symmetry energy. This is strengthening our agreement that elliptical flow depends on the $N / Z$ ratio or alternatively the isospin dependence rather than on the size of the interacting system.

In Fig 5, we display the variation of the excitation function of elliptical flow $v_{2}$ for free, LCP's and IMF's over entire rapidity and mid-rapidity region. The elliptical flow is found to become less positive (entire rapidity) or more negative (mid-rapidity) with the increase in the beam energy, upto a certain energy, and then again becomes more positive or less negative. This is due to the fact that spectators move faster after the $v_{2}$ has reached a minimum value[5]. This energy, at which the behavior changes is found to decrease with the size of 


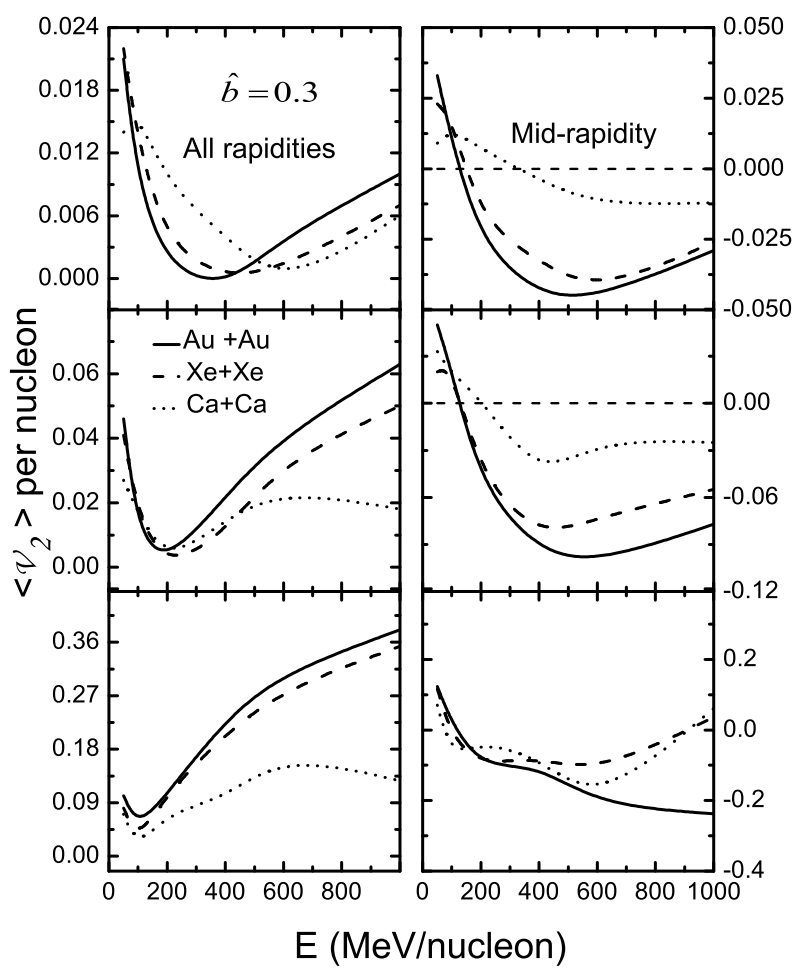

FIG. 5: The variation of the elliptical flow, summed over entire transverse momentum, with beam energy at $\hat{b}=0.3$ for different symmetric reactions over entire rapidity range and at mid-rapidity in the left and right panels, respectively. The top, middle and bottom panels have same meaning as that of Fig, 1.

the fragment. It means that the flow of heavier fragments is larger compared to LCP's/free nucleons at all beam energies. These type of findings are also reported by different authors in Ref. [16]. This is true for entire rapidity region as well as for mid-rapidity region.

The interesting phenomena of transition from in-plane to out-of-plane is observed at midrapidity region [4, 26], while no transition is observed when integrated over entire rapidity region. The energy at which this transition is observed is dubbed as transition energy $\left(E_{\text {Trans }}\right)$. It means that participant zone is responsible for the transition from in-plane to out-of-plane. That's why free particles and LCP's, which originate from the participant zone, are showing a systematic behavior with the beam energy as well as with the composite mass of the system. The elliptical flow for these particles is found to become more negative with the increase in the composite mass of the system. Heavier is the system, more is the Coulomb 


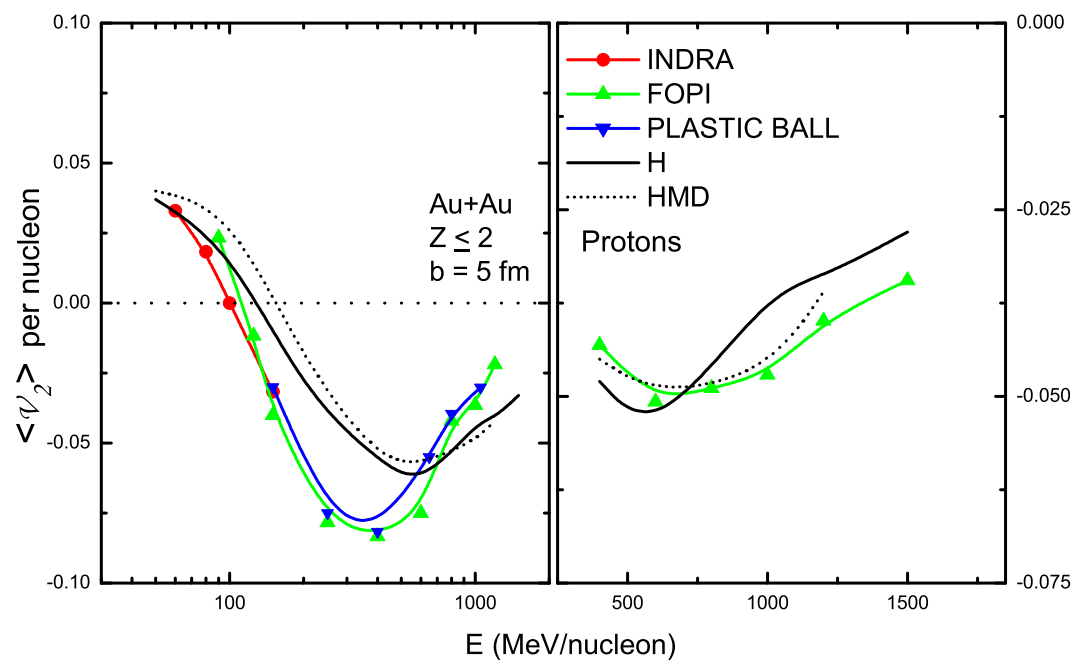

FIG. 6: The variation of the elliptical flow, summed over entire transverse momentum, with beam energy at $|y|=\left|\frac{y_{c . m}}{y_{\text {beam }}}\right| \leq 0.1$ for ${ }_{79} A u^{197}+{ }_{79} A u^{197}$ reaction. Here comparison is shown with experimental findings of INDRA, FOPI and PLASTIC BALL Collaborations [4 $[$ 6].

repulsion and more negative is the elliptical flow. This systematics of $E_{\text {Trans }}$ with composite mass of the system is discussed later.

In Fig. 6, we show $v_{2} @$ mid rapidity $\left(|y|=\left|\frac{y_{c . m}}{y_{\text {beam }}}\right| \leq 0.1\right)$ for $Z \leq 2$ (left panel) and for protons (right panel) as a function of the incident energy. The rapidity cut is in accordance with the experimental findings. The theoretical results are compared with the experimental data extracted by INDRA, FOPI and PLASTIC BALL collaborations [4 6]. With the increase in the incident energy, elliptical flow $v_{2}$ changes from positive to negative values exhibiting a transition from the in-plane to out-of-plane emission of nucleons. This is because of the fact that the mean field, which contributes to the formation of a rotating compound system, becomes less important and the collective expansion process based on the nucleon-nucleon scattering starts to be predominant. This competition between the mean field and N-N collisions depends strongly on the effective interactions that leads to the different transition energy due to different equations of state. Due to repulsive nature of the momentum dependent interactions, which leads to the suppression of binary collisions, less squeeze-out is observed in the presence of momentum dependent interactions (HMD) compared to static one $(\mathrm{H})$. The maximal negative value of $v_{2}$ is obtained around $E=500 \mathrm{MeV} /$ nucleon with 


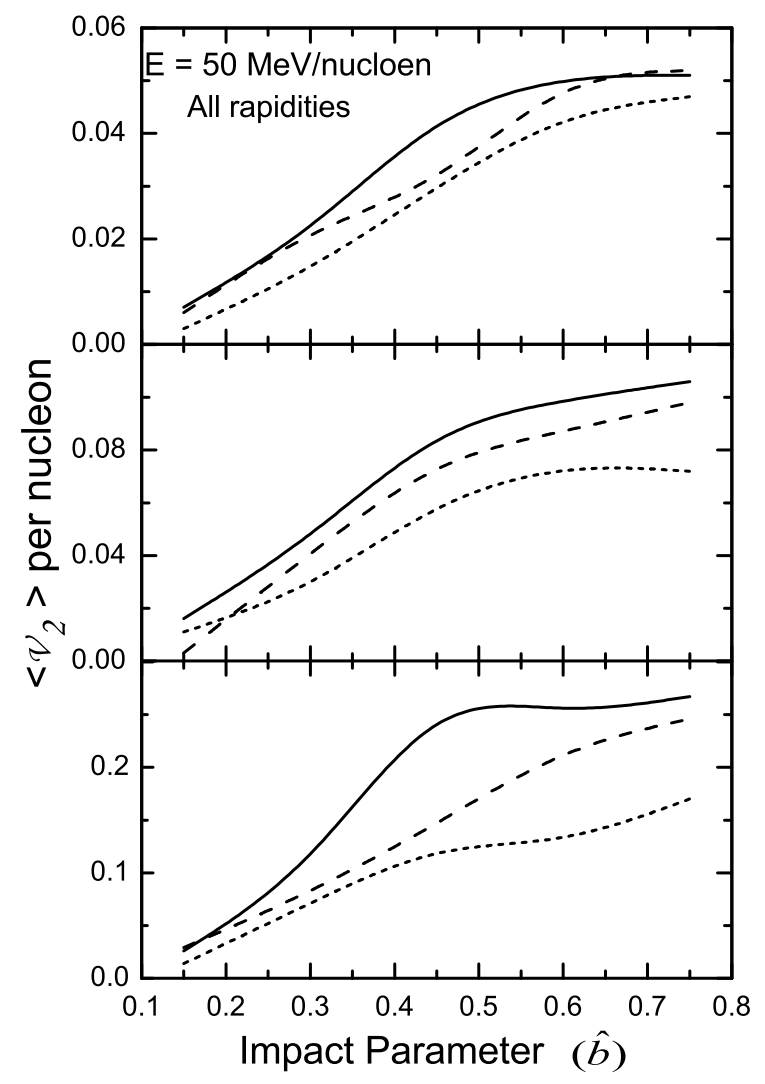

FIG. 7: The impact parameter dependence of the elliptical flow, summed over entire transverse momentum and rapidity distribution, at incident energies $50 \mathrm{MeV} /$ nucleon. The top, middle and bottom panels are for free particles, LCP's and IMF's, respectively.

hard $(\mathrm{H})$ and hard momentum dependent (HMD) equations of state. This out-of-plane emission decreases again towards the higher incident energies. This happens due to faster movement of the spectator matter after $v_{2}$ reaches the maximal negative value [5]. This trend is in agreement with experimental findings. A close agreement with data is obtained in the presence of hard equation of state for $Z \leq 2$ particles, while, in the presence of momentum dependent interactions for protons. Similar results and trends have also been reported by Zhang et.al. in their recent communication [26].

The investigation of the elliptical flow with scaled impact parameter over entire rapidity range is displayed in Fig:7. Here the top, middle and bottom panels represent the free nucleons, LCP's and IMF's. The value of the elliptical flow $v_{2}$ becomes more positive with the impact parameter and composite mass of the system at $E=50 \mathrm{MeV} /$ nucleon, while at 


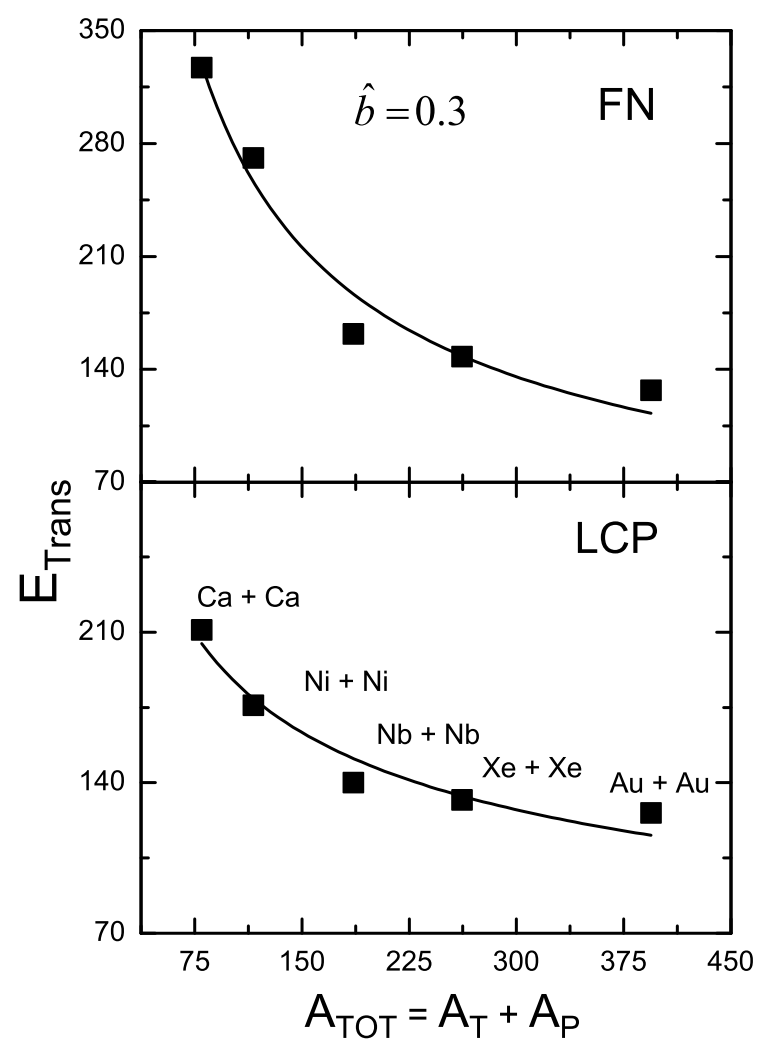

FIG. 8: The transition energies for elliptical flow at intermediate energies as a function of the combined mass of the system. The upper panel is for the free nucleons, while, lower panel is for the LCP's.

higher energies (not shown here), it is found to become less positive (entire rapidity) or more negative (mid-rapidity) with composite mass of the system. This is indicating the dominance of the in-plane flow at low incident energies with increasing impact parameter and composite mass of the system. Moreover, dominance of the out-of-plane flow at higher energies with small impact parameter and composite mass of the system is observed. With the increase in the beam energy, the expansion of the compressed zone becomes more vigorous, while, with an increase in the impact parameter, participant zone decreases, resulting an increases in the spectator region indicating dominance of azimuthal anisotropy with impact parameter. On the other hand, it reduces with beam energy. These observations are consistent with the experimental findings and with other theoretical works [16, 21, 27].

Finally, we carry out the system size dependence of the elliptical flow for free nucleons and 
LCP's. In Fig, 8 , we show the transition energy $E_{\text {Trans }}$ as a function of the composite mass of the system for free nucleons and LCP's. From the figure, we see that the transition energy decreases with the composite mass of the system as well as with the size of the fragment. The reason for this is that the pressure produced by the Coloumb interactions increases with the system size. This dependence can be fitted using a power law of the kind:

$$
E_{\text {Trans }}=C\left(A_{\text {tot }}^{-\tau}\right)
$$

The exponent $\tau$ is found to be 2 times for free particles (0.67) compared to LCP's (0.35). This exponent is quite smaller compared the exponent of balance energy in directed flow [26]. This is due to the different origin of the balance and transition energy. The balance energy counter balances the mean field and N-N collisions, while transition energy is due to the more complex effects such as expansion of the compressed zone and shadowing of the cold spectator matter.

\section{CONCLUSION}

In conclusion, we have investigated the elliptical flow of fragments for different reacting systems at incident energies between 50 and $1000 \mathrm{MeV} /$ nucleon using isospin-dependent quantum molecular dynamics (IQMD) model. The elliptical flow is found to show a transition from in-plane to out-of-plane at a certain beam energy in mid-rapidity region, while no such transition is observed when integrated over entire rapidity region. This transition energy is found to decrease with the composite mass as well as with the size of the fragment. The transition energy is further parametrized in term of mass power law. In addition, LCP's exhibit isospin effect in the mid-rapidity region.

\section{Acknowledgments}

This work has been supported by the Grant no. 03(1062)06/ EMR-II, from the Council of Scientific and Industrial Research (CSIR) New Delhi, Govt. of India. 
[1] P. Danielewicz, R. Lacey, and W. G. Lynch, Science 298, 1592 (2002); H. Stöcker and W. Greiner, Phys. Rep. 137, 277 (1986); W. Reisdorf and H. G. Ritter, Ann. Rev. Nucl. Sci. 47, 663 (1997); C. Hartnack and J. Aichelin, Phys. Rev. C 49, 2801 (1994); S. Kumar, S. Kumar, and R. K. Puri, Phys. Rev. C 78, 064602 (2008); A. R. Raduta and F. Gulminelli, ibid. 75, $024605(2007)$

[2] J. Aichelin, Phys. Rep. 202, 233 (1991).

[3] G. D. Westfall et al., Phys. Rev. Lett. 71, 1986 (1993); M. B. Tsang et al., Phys. Rev. C 53, 1959 (1996); Y. M. Zheng, C. M. Ko, B. A. Li, and B. Zhang, Phys. Rev. Lett. 83, 2534 (1999); A. B. Larionov, W. Cassing, C. Greiner, and U. Mosel, Phys. Rev. C 62, 064611 (2000); B. A. Li, A. T. Sustich, and B. Zhang, ibid. 64, 054604 (2001); C. Alt et al., ibid. 68, $034903(2003)$.

[4] J. Lukasik, G. Auger, and M. L. Begemann-Blaich et al., Phys. Lett. B608, 223 (2005).

[5] A. Andronic et al., Nucl. Phys. A679, 765 (2001); Phys. Lett. B612, 173 (2005).

[6] J. Lukasik, et.al., INDRA Collaborations, Int. Workshop on Multifragmentation and related topics (IWM 2003) Caen, France (2003).

[7] S. Kumar, M. K. Sharma, R. K. Puri, K. P. Singh, and I. M. Govil, Phys. Rev. C 58, 3494 (1998); A. D. Sood, R. K. Puri, and J. Aichelin, Phys. Lett. B594, 260 (2004).

[8] A. D. Sood and R. K. Puri, Phys. Rev. C 69, 054612 (2004); Eur. Phys. A30, 571 (2006).

[9] L. W. Chen and C. M. Ko, Phys. Lett. B634, 205 (2006); Phys. Rev. C 73, 014906 (2006).

[10] M. Gyulassy, K. A. Frankel, and H. Stöcker, Phys. Lett. B110, 185 (1982); P. Danielewicz and M. Gyulassy, Phys. Lett. B129, 283 (1983); C. M. Ko and G. Q. Li, J. Phys. G: Nucl. and Part. 22, 1673 (1996); G. Q. Li, C. M. Ko, and B. A. Li, Phys. Rev. Lett. 74, 235 (1995); G. Q. Li and C. M. Ko, Nucl. Phys. A594, 460 (1995); B. A. Li and C. M. Ko, Phys. Rev. C 52, 2037 (1995); Phys. Rev. C 58, R1382 (1998); Nucl. Phys. A601, 457 (1996).

[11] H. Sorge, Phys. Rev. Lett. 78, 2309 (1997); J. Y. Ollitrault, Phys. Rev. D 46, 229 (1992).

[12] S. Voloshin and Y. Zhang, Z. Phys. C 70, 665 (1996).

[13] C. Hartnack et al., Eur. Phys. J. A1, 151 (1998).

[14] H. Kruse, B. V. Jacak, and H. Stöcker, Phys. Rev. Lett. 54, 289 (1985); J. J. Molitoris and 
H. Stöcker, Phys. Rev. C 32, R346 (1985); J. Aichelin and G. Bertsch, Phys. Rev. C 31, 1730 (1985).

[15] D. J. Magestro, W. Bauer, and G. D. Westfall, Phys. Rev. C 62, 041603(R) (2000); E. Lehmann et al., Z. Phys. A355, 55 (1996); A. D. Sood and R. K. Puri, Phys. Rev. C 73, 067602 (2006); ibid. 70, 034611 (2004).

[16] H. Y. Zhang et al., J. Phys. G: Nucl and Part. 28, 2397 (2002).

[17] E. Lehmann et.al., Prog. Part. Nucl. Phys. 30, 219 (1993); Phys. Rev. C 51, 2113 (1995).

[18] Y. K. Vermani and R. K. Puri, Eur. Phy. Lett. 85, 62001 (2009); Y. K. Vermani. S. Goyal, and R. K. Puri, Phys. Rev. C 79, 064613 (2009); J. Phys. G:Nucl. and Part. in press (2010); S. Kumar and R. K. Puri, Phys. Rev. C 58, 1618 (1998); R. K. Puri and J. Aichelin, J. Comp. Phys. 162, 245 (2000); J. Singh, S. Kumar, and R. K. Puri, Phys. Rev. C 62, 044617 (2000); R. K. Puri, C. Hartnack, and J. Aichelin, Phys. Rev. C 54, R28 (1996).

[19] J. Singh and R. K. Puri, Phys. Rev. C 62, 054602 (2000).

[20] M. Colona, M. D. Toro, G. Ferini, and V. Greco, Catania workshop on nucleon and neutrino astrophysics, 15-16 Feb., (2007); M. Di Toro, S. J. Yennello, and B. A. Li, Eur. Phys. J. A30, $153(2006)$.

[21] Y. G. Ma, W. Q. Shen, J. Feng, and Y. Q. Ma, Phys. Rev. C 48, 1492 (1993); Z. Phys. A 344, 469 (1993); Y. G. Ma, W. Q. Shen, Z. Y. Zhu, Phys. Rev. C 51, 1029 (1995); W. Q. Shen et al., Nucl. Phys. A551, 333 (1993); R. Lacey et al., Phys. Rev. Lett. 70, 1224 (1993).

[22] T. Z. Yan et al., Chin. Phys. 16, 2676 (2007).

[23] F. S. Zhang, L. W. Chen, W. F. Li, and Z. Y. Zhu, Eur. Phys. J. A 9, 149 (2000).

[24] M. B. Tsang et al., Phys. Rev. Lett. 71, 1502 (1993).

[25] L. W. Chen, C. M. Ko, and B. A. Li, Phys. Rev. C 68, 017601 (2003).

[26] Y. Zhang and Z. Li, Phys. Rev. C 74, 014602 (2006).

[27] J. Peter et al., Nucl. Phys. A519, 611 (1990); Z. Y. He et al., ibid. 598, 248 (1996). 